\title{
Time for a Next-Generation Nuclear Medicine Gamma Camera?
}

\author{
I. George Zubal, PhD, Program Director for Nuclear Medicine, CT, and X-Ray, National Institute of Biomedical \\ Imaging and Bioengineering, Bethesda, MD
}

$\mathrm{H}$ al Anger invented the gamma camera in 1957, and it is fair to say that the basic geometry and components of his camera design have remained substantially the same, while its use in general clinical applications has been optimized for imaging 140-keV gamma rays. The past 60 years have seen some improvements in NaI scintillators, readout electronics, collimators, reconstruction algorithms, and image analysis. During a short period in the late 1990s and early 2000s, opposing Anger cameras were used for clinically acquiring positron-emitting isotopes, and some camera components were reengineered for imaging 511$\mathrm{keV}$ coincident photons. Not surprisingly, dedicated PET cameras proved to be the better choice for imaging PET radiotracers.

One clinical application, however, generated substantial camera variations. The highly successful use of cardiac imaging in the United States has spurred interesting new camera designs and novel radiopharmaceuticals. Nuclear cardiology currently represents more than $50 \%$ of all U.S. nuclear medicine scans. Dedicated cardiac cameras have implemented 7-, 9-, and 19-pinhole collimators, early use of new detectors (CsI and CZT), L-shaped camera configurations, and chair-based imaging. Given this important and well-recognized clinical application, camera designs morphed into a variety of geometries, detector materials, and associated reconstruction methods. Whole-body (bone scans) and brainimaging cameras have evolved over these same years, but current whole-body scanners employ a standard Anger camera translated along the patient bed. Dedicated brain cameras have not yet achieved broad acceptance and, perhaps, are awaiting new breakthroughs in theranostic applications for brain imaging.

Recent developments in unsealed source therapies using electron- and $\alpha$-emitting radiopharmaceuticals would benefit from improvements in patient-specific dosimetry estimates. ${ }^{177} \mathrm{Lu},{ }^{90} \mathrm{Y}$, and ${ }^{223} \mathrm{Ra}$ are the most common isotopes currently used to deliver high doses to the targeted cancer and to spare healthy tissue. Because of the high radiation doses delivered locally by these radiotherapeutic agents, it is important to know the patient-specific uptake distribution of these ligands. Analogs of these ligands have been developed to assess uptakes. By imaging the analog (labeled with ${ }^{68} \mathrm{Ga}$, for example), one assumes that the analog has the same pharmacodynamics and pharmacokinetics as the ${ }^{90} \mathrm{Y}$ - or ${ }^{177} \mathrm{Lu}$-labeled therapy ligand. Such an assumption becomes complicated with ${ }^{223} \mathrm{Ra}$, where such a process would be ignoring the doses to healthy tissues delivered by ${ }^{223} \mathrm{Ra}$ daughters.
Imaging of these radiotherapy ligands has been investigated. Two of the 6 photopeaks (113 and $208 \mathrm{keV}$ ) of ${ }^{177} \mathrm{Lu}$ were imaged with additional energy windows set to subtract scatter from higher energy emissions (1). An array of bremsstrahlung emissions, together with internal pairproduction annihilation radiation, was used to produce ${ }^{90} \mathrm{Y}$ images (2). Images of ${ }^{223} \mathrm{Ra}$ (and its daughter ${ }^{219} \mathrm{Rn}$ ) were acquired using 3 photopeaks $(85,154$, and $270 \mathrm{keV}$ ) with 3 additional windows to deal with scattered events (3). Imaging protocols become more complicated for other $\alpha$ emitters, including ${ }^{225} \mathrm{Ac},{ }^{211} \mathrm{At},{ }^{212} \mathrm{~Pb}$, and others yet to be considered for therapy. These isotopes pose a challenge to nuclear medicine camera systems because the radiations lie outside current clinical imaging protocols. New camera designs could lead to improved image quantitation.

Is it time to reconsider the instrumentation we use for theranostic methods for these $\alpha$-emitting unsealed sources? If nuclear cardiology could develop an array of specialized camera designs and acquisition methods to specifically image the heart, can we consider new instrumentation and image analysis methods that would give us improved insights into targeted cancer therapy? The next phases of therapy outcomes that use these new ligands will speak to this question. A dedicated therapy camera could help to maximize dose to the cancer and minimize dose to healthy tissues. It seems axiomatic that by improving methods for imaging these new ligands, we would improve the success of the clinical therapy outcomes. This tandem step forward appears reasonable.

To which ideas can we turn for meeting this imaging challenge? The gamma emissions of these new therapy isotopes are often low yield. Can previous work on highsensitivity coded apertures or Compton cameras be reinvestigated for some of the higher energy emissions? Can gas electron multiplication detectors be used to measure gamma rays and their incident angles without the use of collimators $(4,5)$ ? New detector systems have been and are being developed by PET instrumentation investigators, with some promising coincidence timing approaching 1 picosecond. Can any of these detectors be reapplied for single-photon imaging? Because some of the $\alpha$ emitters (despite the low yield of individual gamma emissions) emit many $10 \mathrm{~s}$ of gammas at various energies (e.g., ${ }^{225} \mathrm{Ac}$ ), could very high-energy resolution detectors be used to acquire the various gammas by picking out these photopeaks (and rejecting most other scattered photons) to assemble an image of unscattered multienergy gammas? Can recent advances in deep learning play an important role in imaging and estimating patient dose?

(Continued on page $17 N$ ) 
$\mathbf{T}$ he National Council on Radiation Protection and Measurements (NCRP) in May issued a new report on Evaluating and Communicating Radiation Risks for Studies Involving Human Subjects: Guidance for Researchers and Institutional Review Boards (Report No. 185). The report was developed by an NCRP scientific committee chaired by Julie Timins, MD, an experienced diagnostic radiologist board certified in general radiology and nuclear medicine, who is also chair of the New Jersey Commission on Radiation Protection. In an executive summary, the report's authors noted that the extent of knowledge about ionizing radiation in medical procedures and potential adverse effects varies substantially among members of the public and within the medical community. This variation is also seen in guidelines used across academic and other institutions for the conduct of human research involving radiation. The report is intended to address the need for "comprehensive, consistent, and accurate guidance on radiation risks of research protocols that involve the use of ionizing radiation to those who develop protocols and conduct research involving human subjects and to institutional review boards (IRBs) that review these protocols." In a release accompanying the publication, NCRP said that the report seeks to fill existing guidance gaps by: (1) providing basic information about ionizing radiation and radiation biology, including medical imaging and treatments that involve radiation; (2) identifying the governmental agencies that oversee research and radiation; (3) citing the relevant regulatory requirements; (4) providing guidance regarding the estimation of radiation dose and risk in research protocols; (5) discussing ethical considerations involved in human studies research; and (6) presenting in detail the requirements for ensuring and obtaining truly informed consent.

The comprehensive document has specific value for research staff, IRBs, and other research review entities that involve personnel who may have limited backgrounds in radiation science. For these individuals, the report is intended "to help researchers optimize radiation use in research protocols, IRBs to perform due diligence in review of those protocols, and to promote understanding of the potential short- and long-term health effects" by providing historical and regulatory background, definitions, descriptions of medical imaging studies and procedures, and more than 500 reference sources. The report covers information needed for research protocol development and evaluation, including basic information on radiobiology, radiation protection, and metrics pertinent to radiation; regulatory requirements for the conduct and supervision of research; in-depth discussions on estimation of radiation dose and risk and the appropriate use of effective and absorbed dose; ethical principles relevant to human studies research involving radiation exposure, including those unique to vulnerable populations, including children; and the informed consent process and examples of language to assist in developing informed consent documents. These examples include "plain language" suggestions to simplify and clarify protocols for participants.

The report is available for purchase from NCRP at https:// ncrponline.org/shop/reports/report-no-185-evaluating-andcommunicating-radiation-risks-for-studies-involving-humansubjects-guidance-for-researchers-and-institutional-reviewboards-2020/. Members of the American Association of Physicists in Medicine may download the document at no charge at https://www.aapm.org/pubs/ncrp/detail.asp?docid=185.

\section{(Continued from page 16N)}

These and other questions will be considered and discussed at a National Institute of Biomedical Imaging and Bioengineering (NIBIB) workshop on "Engineering New Instrumentation for Imaging Unsealed Source Radiotherapy Agents," to be held August 17 and 18 at the Natcher Center on the main National Institutes of Health (NIH) campus in Bethesda, MD. We believe that such discussions are timely for moving hand-in-hand into the testing and use of $\alpha$-emitting therapy trials. The mission of NIH's NIBIB is to improve health by leading the development and accelerating the application of biomedical technologies. Among the many technologies supported, NIBIB researchers believe the challenge of considering cameras that would deliver improved dosimetry measurements for optimizing the outcome of $\alpha$-emitting radiotherapy ligands is one that merits a serious look. For more information on the workshop, see https://www.imagingtherapy.nibib.nih.gov/.

\section{REFERENCES}

1. Sanders JC, Kuwert T, Hornegger J, Ritt P. Quantitative SPECT/CT imaging of Lu-177 with in vivo validation in patients undergoing peptide receptor radionuclide therapy. Mol Imaging Biol. 2015;17(4):585-593.

2. Wright CL, Zhang J, Tweedle MF, Knopp MV, Hall NC. Theranostic imaging of yttrium-90. Biomed Res Int. 2015;2015:481279.

3. Benabdallah N, Bernardini M, Bianciardi M, de Labriolle-Vaylet C, Franck D, Desbrée A. ${ }^{223}$ Ra-dichloride therapy of bone metastasis: Optimization of SPECT images for quantification. EJNMMI Res. 2019;9(1):20.

4. Sauli F. The gas electron multiplier (GEM): Operating principles and applications. Nucl Instrum Methods Phys Res A. 2016;805(1):2-24.

5. Brunbauer FM, Lupberger M, Oliveri et al. Radiation imaging with optically read out GEM-based detectors. J Instrum. 2018;13:T02006. Available at: https://iopscience. iop.org/article/10.1088/1748-0221/13/02/T02006/pdf. 